\title{
A Model of Nova Delphini 1967 from its Nebular Spectrum
}

\author{
WALTRAUT CAROLA SEITTER (Northampton, Bonn)
}

Nova Delphini 1967 is outstanding among other recent novae since it belongs to the rare class of extremely slow objects. Another unusual characteristic is the pronounced pattern of the emission lines during the nebular stage.

These profiles permit a simple and quick analysis of the structure and relative physical conditions within different parts of the expanding nova envelope without extensive intensity and radial velocity determinations - though a more thorough analysis using the latter data is in progress.

Sixteen spectrograms of Nova Delphini 1967 (HR Del) were obtained between November 1968 and September 1970, that is during the nebular stage of the nova. E. GEYER and G. CHINCARINI worked with the Cassegrain spectrograph of the $42^{\prime \prime}$-telescope at the Hoher List Observatory of Bonn University using reciprocal linear dispersions between 29 and $86 \mathrm{~A} / \mathrm{mm}$ on blue sensitive plates and $136 \mathrm{~A} / \mathrm{mm}$ on red and infrared plates.

They kindly put their observational material at my disposal.

\section{Radial Velocities}

On the largest dispersion spectrograms the emission lines show six brightness maxima (Fig. 1) which are interpreted as individual narrow emission components of different radial velocities. In the smaller dispersion spectrograms the least pronounced peaks - which are the ones with intermediate radial velocities - can no longer be differentiated, the central peaks sometimes appear as a single feature.

Radial velocity measurements for [O III] 4363 on a high dispersion plate taken in December 1968 indicate a nearly symmetrical distribution of the components with respect to the emission center, with radial velocities corresponding very roughly to $\pm 100, \pm 300$ and $\pm 500 \mathrm{~km} / \mathrm{sec}$, referred to the emission center.

Detailed radial velocity measurements permitting the determination of radial velocity variations as a function of the emitting ion and as a function of time are not yet completed. A preliminary inspection indicates somewhat larger broadening for some lines, in particular Fe VII 6087. However, on the basis of the general appearance of the spectra it may tentatively be assumed that there are no major variations and that the central components have velocities near $\pm 100 \mathrm{~km} / \mathrm{sec}$ and the predominant outer components velocities near $\pm 500 \mathrm{~km} / \mathrm{sec}$. A discussion of the intermediate components is omitted in order to simplify the procedure.

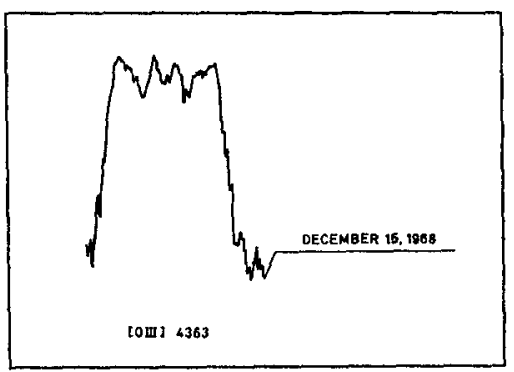

Fig. 1 


\section{Relative Intensitles}

The most remarkable properties of the emission features are the systematic intensity differences between the two central and the two outer components as a function of ionization energy, transition probability and time.

This can be interpreted as a consequence of the different physical conditions of those parts of the expanding nova envelope where the central components originate and those parts where the outer components are emitted. In the following the relative intensities of the central and outer components will be used to derive the relative physical conditions in the regions of their origin.

Table 1 summarizes the elements and ions which are represented in the spectra with sufficiently strong lines.

Table 1

\begin{tabular}{|c|c|}
\hline $\mathrm{H}$ & Balmer decrement \\
\hline $\begin{array}{l}\mathrm{HeI} \\
\mathrm{He} \text { II }\end{array}$ & $\begin{array}{l}\text { decrement } \\
\text { ionzation energy }\end{array}$ \\
\hline \multicolumn{2}{|l|}{$\begin{array}{l}\text { C II } \\
\text { C III }\end{array}$} \\
\hline $\begin{array}{l}\text { N II } \\
\text { N III } \\
\text { N IV } \\
\text { N V } \\
\text { [N II] }\end{array}$ & $\begin{array}{l}\text { ionzation energy } \\
\text { transition probabilities (auroral versus nebular) }\end{array}$ \\
\hline $\begin{array}{l}\text { O I } \\
\text { O II } \\
\text { O III } \\
{\left[\begin{array}{ll}O \\
{[O}\end{array}\right]} \\
{[O \text { II] }} \\
\end{array}$ & $\begin{array}{l}\text { fluorescence } \\
\text { ionzation energy } \\
\text { transition probability } \\
\text { transition probabilities (auroral versus nebular) }\end{array}$ \\
\hline [Ne III] & transition probability \\
\hline [S II] & transition probability \\
\hline $\begin{array}{l}\mathrm{Fe} I I \\
{[\mathrm{Fe} I I]} \\
{[\mathrm{Fe} \mathrm{VII]}}\end{array}$ & ionzation energy \\
\hline
\end{tabular}

$O I$ is the only exception since information concerning the structure of $O$ I 8446 was obtained from a paper by ANDRILLAT and HOUZIAUX (1). The table also indicates which particular aspect of the transition is considered in the discussion; no comment means insufficient evidence for the solution of the present problem.

All of the following arguments are illustrated by density tracings of emission lines from plates taken in 1968 and 1969 . For the sake of clarity, only spectra of equal or nearly equal dispersions are used. The general tendencies, however, can be followed on all spectrograms including the ones not shown here.

First we shall trace lines from ions requiring high ionization or excitation energies.

The central components of He II 4686 are considerably weaker than the outer components throughout the observing period (Fig. 2). The upper level of He II 4686 has an excitation energy of $50.80 \mathrm{eV}$. 


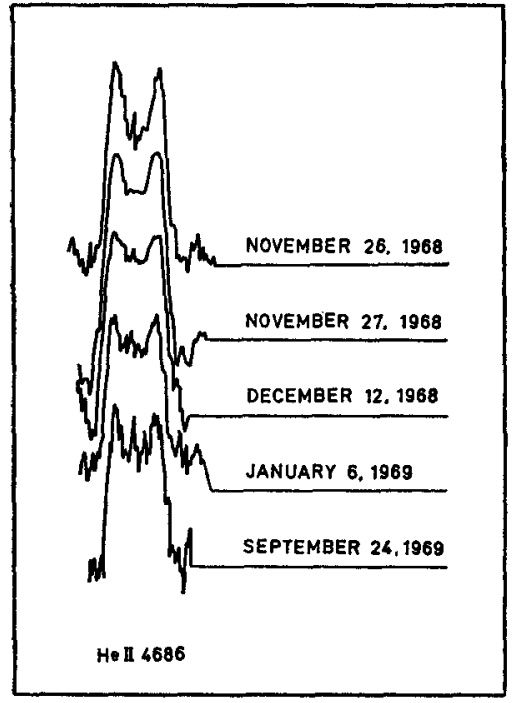

Fig. 2

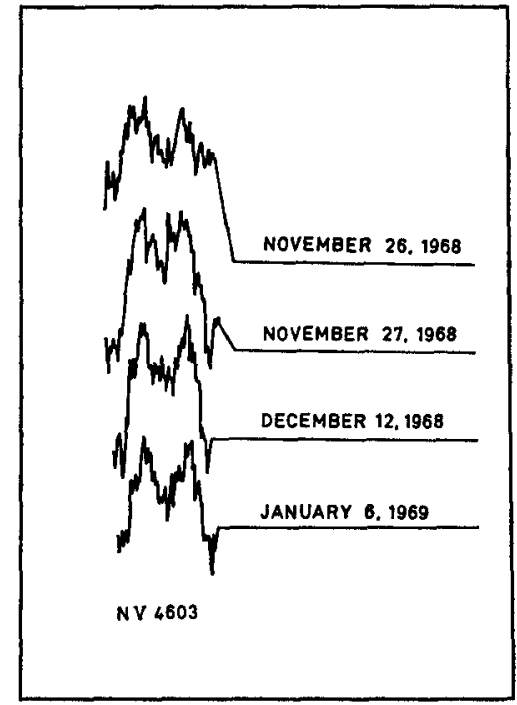

Fig. 3

For N V 4603 the intensity pattern is the same as that of the He II emissions (Fig. 3). To ionize nitrogen four times an energy of $77.09 \mathrm{eV}$ is needed.

The forbidden line of Fe VII at $6087 \AA$ shows the like behaviour (Fig. 4). The neccessary ionization energy is of the order of $100 \mathrm{eV}$.

While some other lines show central dips at certain times, only the above mentioned lines - and possibly O III 3757 -, which require high energies, show the same structure throughout the time of observation. It is therefore concluded:

The ionization temperature of those regions of the nova envelope where the outer components originate is higher than the temperature of those parts where the central components originate.

In the second part of the present discussion the relative emission intensities of forbidden lines - with the exception of the already discussed forbidden FeVII lines - will be considered.

The nebular lines [O III] 5007, 4959 (lower transition probabilities) have stronger central components, the auroral line at $4363 \AA$ (higher transition probability) shows equal strength of all components in the earlier spectra, lower strength for the central components in the later spectra (Fig. 5).

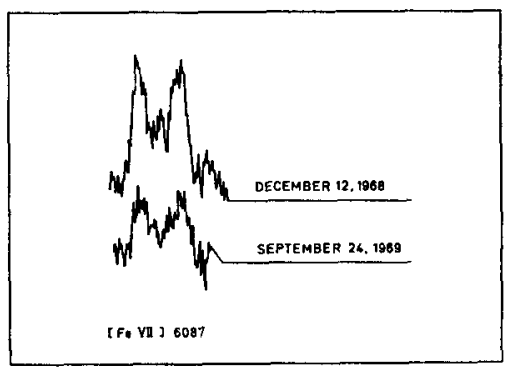

Fig. 4 


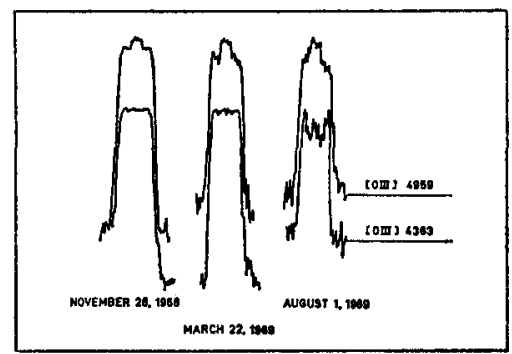

Fig. 5

The auroral line at $5755 \AA$ is the only unblended line of [N II]. It shows a behaviour similar to that of the auroral line of [O III]. For the nebular [N II] line at $6584 \AA$ a central dip cannot be excluded in the earlier spectra but a central peak is indicated in the later ones. Definite statements are not possible on account of the strong superimposed $\mathrm{H} \alpha$ emission. The nebular line at $3869 \AA$ is the only [Ne III] line clearly visible. It shows the same structure as the nebular lines of [O III] (Fig. 6).

Conclusion: Since the electron temperature and/or electron density is higher for regions with large ratios I (auroral)/I (nebular), the regions where the outer components originate must be denser andlor hotter than the regions of origin of the central components.

It is interesting to note an exception among the nebular lines not unlike the one possibly indicated for [N II] 6584 : [O I] 6300 (the line at $6364 \AA$ is blended) is in the earlier spectra stronger in the outer components and conforms to the pattern of the highly forbidden O III and Ne III lines only as late as July 1969 (Fig. 7). No explanation is offered for these deviations.

The profile of OI 8446 (taken in August 1968), which also shows stronger outer components, is easily explained in terms of the Bowen fluorescence mechanism. It accounts for an enhancement of the $\mathcal{O} I$ emission in regions of strong Lyman $\beta$ absorption.

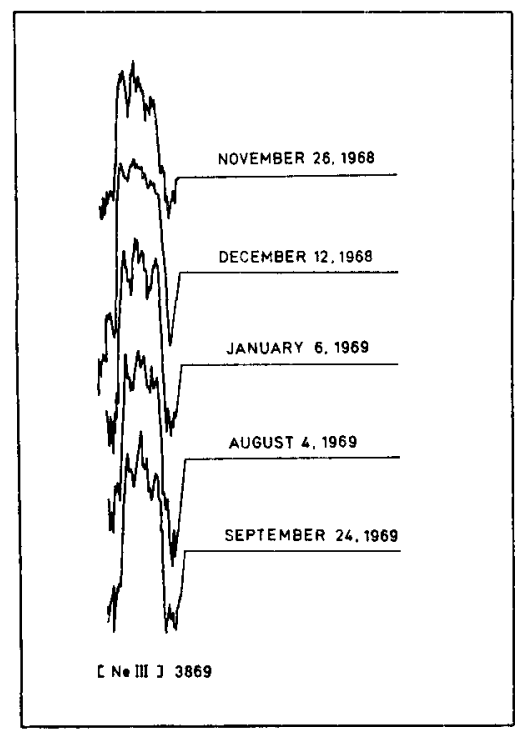

Fig. 6 


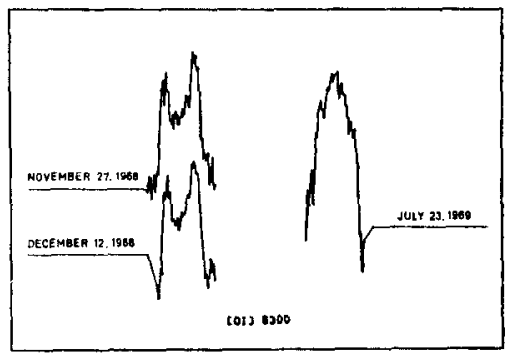

Fig. 7

Strong Lyman $\beta$ absorption is in good agreement with the existence of an optically thick shell.

The final part of the discussion of line intensities is devoted to the study of the Balmer and helium decrements. Fig. 8 shows the intensity differences between the central and outer components of $\mathrm{H} \beta$ and $\mathrm{H} \varepsilon$ at two different times. The lengths of the arrows are arbitrary; the relative lengths, however, indicate the relative steepness of the decrements of the central and outer components.

It is seen that: the central components have a flatter decrement indicating an optically thinner shell. The difference in flatness seems to increase with time. If a general decrease in density for both parts of the expanding envelope is assumed, this means that the parts where the central components originate show a faster density decrease.

The helium decrement is illustrated by the two diffuse triplet lines He I 5876 and HeI 4471 (Fig. 9). The decrement is again flatter for the central and steeper for the outer components indicating a higher optical thidkness in $\lambda 3889$ for the region of origin of the outer components.

It must be remembered, however, that this decrement is not a very sharp indicator of optical depth. Mudh larger differences are found for the diffuse singlet line Hel 7065 when compared with He 5876 in the sense that here the flatter decrement indicates the larger optical depth. Both of these lines are found together on only two of our spectra so that the evidence is rather marginal. They seem to verify the conclusion drawn from the triplet ratios.

All neutral helium lines appear to be more irregular in their behaviour than lines of other elements so that any statement must be taken with care. At times the reverse of the above described situation cannot be excluded.

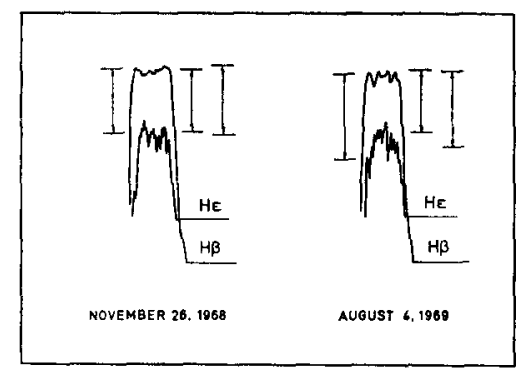

Fig. 8 


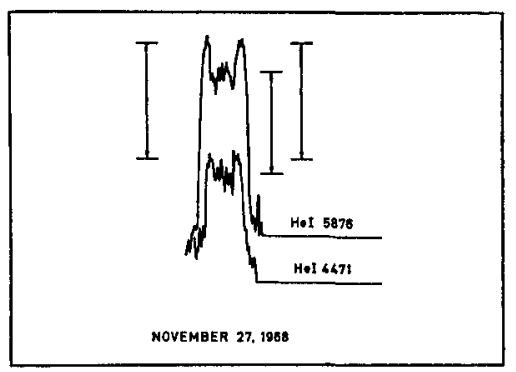

Fig. 9

\section{The Physical Nova Model}

We summarize: Fairly consistent evidence from lines of different ions points to the fact that the nova envelope has two physically distinct regions of which the one emitting the line components of smaller positive and negative velocities is cooler and less dense than the one where the larger radial velocity components originate.

\section{The Geometrical Nova Model}

While the above result permits several solutions for the geometrical structure of the nova envelope, the most likely seems to be one which agrees with the structure of previously observed nova shells. MUSTEL and BOYARCHUK (2) derived from photographs of actual nebulae around DQ Herculis and V603 Aquilae a simple model consisting of an equatorial ring, possible secondary rings at higher latitudes and two polar blobs ejected from the nova. The blobs are strong (for DQ Her) on plates sensitive to the O III nebular lines, the ring is strong in the spectral region around $\mathrm{H} \alpha$ and the two nebular $\mathrm{N}$ II lines. The orbital planes of the system binaries are coincident with the planes of the equatorial rings.

It is obvious that the regions corresponding to the smaller radial velocities on our spectrograms show properties comparable to the blobs while the equatorial ring can be considered to produce the components of a larger radial velocity.

It has been computed, see e. g. PAYNE-GAPOSCHKIN (3), that an equatorial ring seen nearly edgewise produces strong outer components with a central dip. If the two narrow components arising from the blobs are superimposed we find indeed the observed line structure.

A remaining question is the orientation of the nova shell with respect to our line of sight. If the ring results from the principal nova ejection which occurred at a velocity of nearly $600 \mathrm{~km} / \mathrm{sec}$, we deduce an inclination of about $30^{\circ}$ between the orbital plane and the line of sight. If the blobs are ejected with the velocity of the principal absorption system of the nova or a slightly larger velocity, the angle of inclination must be $8-10^{\circ}$, in order to account for the radial velocity components of $\pm 100 \mathrm{~km} / \mathrm{sec}$. In any case it seems not unreasonable to expect an eclipse if this nova as others has a binary nature.

\section{The Question of an Eclipse}

The final question is then: Is an eclipse already evident from pre-outburst variations of the nova?

HERCZEG (4) has shown in the case of DQ Herculis that an analysis of patrol plate data may indicate an eclipse when the period of the system is already known. The difficulty in the present case is that no period is as yet available.

Dr. WENZEL (5) from the Sonneberg Observatory has kindly made available all preoutburst magnitudes of the nova as estimated from Sonneberg survey plates, a total of 218 values. The majority of the data is spread over such large time intervals that uncertainties in 


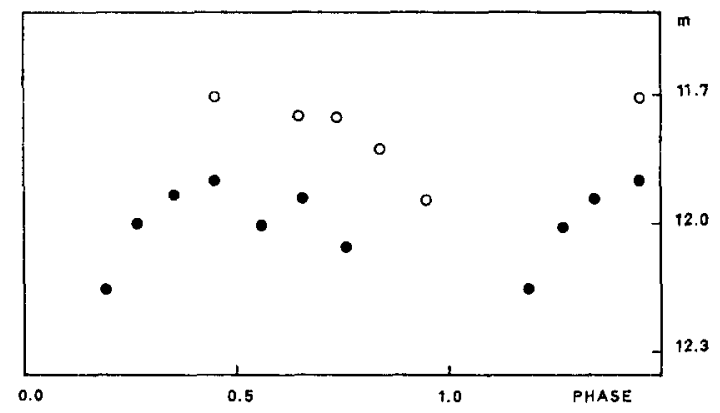

Fig. 10

the period would hopelessly confuse the light curve. A set of 28 magnitude estimates from plates taken within only 26 days seems to be the most promising part of the material. The reductions were made in two years.

First a likely period of 0.5085 was derived from an inspection of the data and the phases were determined.

Secondly, trial runs were made with a number of arbitrary periods between 0.10 and 0.90 days. A somewhat disturbing fact is, that numerous different periods - including those where one must expect a smearing out of the minima due to an accumulation of errors over too many periods - show indications of minima. One of the best looking light curves was obtained from a period around 0.5 days. This is in good agreement with the light curve obtained by the first method.

A confirmation of the first light curve is furthermore the agreement with a light curve derived from data collected during a 62 day time interval beginning 74 days before the 26 day time interval used in the original derivation - except that the earlier magnitudes are consistently fainter. The two light curves derived for a period of $0 \$ 5085$ are given in Fig. 10. All points are averages over $1 / 10$ of the period.

It is obvious that one must wait for the brightness oscillations of the nova to die down before precise magnitude measurements can be made and the question of an eclipse and its period be answered. Until then the present model, derived with such simple means, is suggested as a hypothesis.

$$
\text { References: }
$$

ANDRILLAT, Y., and HOUZIAUX, L., 1969, in "Mass Loss from Stars“, ed. by M. Hack, p. 281.

MUSTEL, E. R. and BOYARCHUK, A. A., 1970, Astrophys. and Space Sc. 6, p. 183.

PAYNE-GAPOSCHKIN, C., 1964, "The Galactic Novae“, p. 62 (Dover).

HERCZEG, T., 1958, Memorie Soc. Astr. Ital. Vol. XXIX, p. 1.

WENZEL, W., 1971, private communication.

\section{Discussion to the paper of SEITTER}

ROSINO: I should like to mention that the splitting of forbidden lines in novae, during the nebular stage, has been observed in most of the novae, besides Nova Delphini. - It seems to be the rule, rather than an exception.

WARNER: I observed Nova Del last year when it was about $2.5 \mathrm{mag}$ above the pre-outburst magnitude, and it did not show any flickering. Observations a few weeks ago show that it is now only about 1 mag. above minimum and is now flickering. I have three runs each of about 2 hrs. duration - there are no signs of an eclipse. This data cannot of course exclude a half day eclipsing period.

HUTCHINGS: The velocity of $500 \mathrm{~km} / \mathrm{sec}$. is not unique in the outburst observations. The choice of a lower one for the ring may help. - From the point of view of computing profiles, the model derived by Dr. SEITTER agreed closely with one of three possible configurations I have found. 